\title{
Ibuprofen versus placebo effect on acute kidney injury in ultramarathons: a randomised controlled trial
}

\author{
Grant S Lipman, ${ }^{1}$ Kate Shea, ${ }^{1}$ Mark Christensen, ${ }_{1}{ }^{C}$ Caleb Phillips, ${ }^{2}$ Patrick Burns, ${ }^{3}$ \\ Rebecca Higbee, ${ }^{4}$ Viktoria Koskenoja ${ }_{1}^{5}$ Kurt Eifling, ${ }^{6}$ Brian J Krabak ${ }^{7}$
}

${ }^{1}$ Department of Emergency Medicine, Stanford University School of Medicine, Stanford, California, USA

${ }^{2}$ Department of Computational Sciences, University of Colorado at Boulder, Boulder, Colorado, USA

${ }^{3}$ Emergency Medicine Residency, University of Washington,

Seattle, USA

${ }^{4}$ Stanford-Kaiser Emergency Medicine Residency, Stanford, USA

${ }^{5}$ Harvard Affiliated Emergency Medicine Residency, Harvard Medical School, Boston, USA ${ }^{6}$ Washington University in St. Louis Emergency Medicine Residency, St. Louis, USA ${ }^{7}$ Department of Orthopedics and Sports Medicine, University of Washington, Seattle, USA

\section{Correspondence to}

Dr Grant S Lipman, Department of Emergency Medicine, Stanford University School of Medicine, 900 Welch Road, Stanford; grantlip@hotmail.com

Received 14 September 2016 Revised 30 May 2017 Accepted 1 June 2017 Published Online First 5 July 2017

\section{ABSTRACT}

Background Despite concerns that non-steroidal antiinflammatory drugs (NSAIDs) contribute to acute kidney injury (AKI), up to $75 \%$ of ultramarathon runners ingest these during competition. The effect of NSAID on AKI incidence in ultramarathon runners is unclear.

Methods Multisite randomised double-blind placebocontrolled trial in the Gobi, Atacama, Ecuador and Sri Lankan deserts to determine whether ibuprofen (400 mg every 4 hours) would be non-inferior to placebo during a 50 -mile $(80 \mathrm{~km})$ foot race. The primary outcome was incidence of AKI defined as severity categories of 'risk' of injury of $1.5 \times$ baseline creatinine $(\mathrm{Cr})$ or 'injury' as $2 \times \mathrm{Cr}_{\text {, }}$ combined to calculate total incidence at the finish line. Non-inferiority margin for difference in AKI rates was defined as $15 \%$.

Results Eighty-nine participants (47\% ibuprofen and $53 \%$ placebo) were enrolled with similar demographics between groups. The overall incidence of AKI was $44 \%$. Intent-to-treat analysis found 22 (52\%) ibuprofen versus 16 (34\%) placebo users developed AKI (18\% difference, $95 \% \mathrm{Cl}-4 \%$ to $41 \%$; OR 2.1, 95\% Cl 0.9 to 5.1 ) with a number needed to harm of 5.5 . Greater severity of AKI was seen with ibuprofen compared with placebo (risk $=38 \%$ vs $26 \% ; 95 \% \mathrm{Cl}-9 \%$ to $34 \%$; injury $=14 \%$ vs $9 \% ; 95 \% \mathrm{Cl}-10 \%$ to $21 \%$ ). Slower finishers were less likely to encounter AKI (OR 0.67, 95\% CI 0.47 to 0.98 ) and greater weight loss (-1.3\%) increased AKI (OR 1.24, $95 \% \mathrm{Cl} 1.00$ to 1.63$)$.

Conclusion There were increased rates of AKI in those who took ibuprofen, and although not statistically inferior to placebo by a small margin, there was a number needed to harm of 5.5 people to cause 1 case of AKI. Consideration should therefore be taken before ingesting NSAID during endurance running as it could exacerbate renal injury.

Trial registration number NCT02272725.

\section{INTRODUCTION}

Ultramarathon foot races, any distance greater than the standard marathon, have increased in popularity worldwide, with $10 \%$ increase per year to 1357 races in 2015, with $>70000$ annual finishers. ${ }^{1}$ Renal dysfunction is a common finding in endurance runners, with acute kidney injury (AKI) observed in $34 \%-85 \%$ of ultramarathoners. ${ }^{2-4}$

The majority of AKI in ultramarathon runners appears to resolve spontaneously, ${ }^{56}$ although it has the potential to progress to acute renal failure and hospitalisation. $^{78}$ Contributing factors to AKI in

\section{Key messages}

What is already known on this subject?

- Acute kidney injury (AKI) is a common finding in endurance runners, encountered in $34 \%-85 \%$ of ultramarathoners.

- Non-steroidal anti-inflammatory drugs (NSAIDs) are thought to contribute to AKI, although $35 \%-75 \%$ of ultramarathoners ingest them during competition.

- Evidence from retrospective studies of NSAID and AKI offers conflicting results, and while the majority show no ill effect, a randomised controlled trial has not fully explored this causative relationship.

\section{What this study adds?}

- This double-blind randomised placebocontrolled trial showed increased rates of AKI in those who took ibuprofen, and although not statistically inferior to placebo by a small margin, there was a number needed to harm of 5.5 people to cause 1 case of AKI.

- Consideration should be taken before ingesting NSAID during endurance running as it could exacerbate renal injury.

- AKI was associated with faster finishers and greater weight loss, suggesting that dehydration may play a role.

this population include myoglobinaemia, increased sympathetic tone, dehydration and reduced renal perfusion from non-steroidal anti-inflammatory drugs (NSAIDs). ${ }^{9}$ A large proportion of athletes take NSAID for their analgesic effects, with 35\%-75\% of ultramarathon runners found to ingest NSAID during competition. ${ }^{10} 11$ Despite their popularity, the benefits of NSAID use are unclear and there is little evidence to support their role in preventing exercise-induced muscle damage, perceived exertion or immediate post-race muscle soreness. ${ }^{10}$

Previous investigations into the role of NSAID as a causative factor for renal injury in endurance runners reveal mixed conclusions. One study found higher serum creatinine $(\mathrm{Cr})$ levels in runners who ingested NSAID ${ }^{8}$ while the majority of studies showed no significant association. ${ }^{3410} 12$ The only prospective trial to date that examined this relationship found no renal dysfunction in the NSAID 
group of runners, but due to methodological constraints its conclusions were limited. ${ }^{12}$ To determine the effect of NSAID on AKI, we compared renal function among ultramarathon runners who were randomised to either ibuprofen or placebo during the course of a 50 -mile $(80 \mathrm{~km})$ foot race. We hypothesised that ibuprofen would not result in a significantly increased rate of AKI compared with placebo.

\section{METHODS}

\section{Design and setting}

We conducted a multisite, randomised, placebo-controlled, double-blind non-inferiority trial during the 50 -mile $(80 \mathrm{~km})$ fifth stage of the RacingThePlanet 7-day, six-stage 155 mile $(250 \mathrm{~km})$ ultramarathons.

\section{Study population}

Eligible participants were between 18 and 75 years of age who were enrolled at the 2015 Atacama Desert, Chile, Gobi Desert, China, Ecuadorian, and 2016 Sri Lankan ultramarathons. These events are run through wilderness terrain with few roads and varying topography. Participants carry all their personal items for the duration of the race, including gear, food (minimum of 2000 calories/day) and clothing. All participants are offered the same amount of water each day $(1.5 \mathrm{~L}$ per $10-12 \mathrm{~km}$ along the course of the stage), with a given allotment of time to complete each stage. These events comprised six stages run over 7 days, with four consecutive single-day stages of 25 miles $(40 \mathrm{~km})$, with a 2-day fifth stage of 50 miles $(80 \mathrm{~km})$, and a final single-day 5-6-mile $(10 \mathrm{~km})$ stage. Eligible participants had to understand English and be registered to run a RacingThePlanet event. Exclusion criteria included ingestion of NSAID or corticosteroids 12 hours prior to stage 5 start, allergy to NSAID, pregnancy or suspected pregnancy and having one kidney or other known medical problem contraindicating NSAID use. The study was approved by Stanford University School of Medicine institutional review board.

\section{Allocation}

Protocol training was by study manual on procedures for blood collection and point-of-care analysis. Participants were assigned in a 1:1 ratio using computer-generated block randomisation. Medications were prepared by Advantage Pharmaceuticals (Rocklin, California, USA), and then packaged and randomised using a computer-generated random sequence by the investigational pharmacy at Stanford University Hospital. Randomisation and drug allocation occurred just prior to the start of the fifth stage.

\section{Study interventions}

Study participants were randomised to either ibuprofen (400 mg) or visually identical placebo taken every 4 hours after the start of the fifth stage for the duration of the stage. As approximately $80 \%$ of runner historically complete a RacingThePlanet 50-mile stage in $<19$ hours, the upper quartile of ingested ibuprofen was estimated to be $1600 \mathrm{mg}$.

We gathered informed consent and baseline demographics the day prior to the race start at race registration, with randomisation prior to the start of the fifth stage. Participants were weighed just before the start of the 50 -mile $(80 \mathrm{~km})$ stage 5 with no pre-race phlebotomy. Each participant was weighed in running gear and shoes without a backpack, using a calibrated battery-operated digital scale (SC-505 HoMedics; Commerce Tsp, Michigan, USA) placed on a level surface. At the finish line of stage 5 , or on removal from competition, weights were measured by the same procedure and fingertip blood samples obtained via lancet and capillary collection tube, then analysed immediately after collection using an iSTAT point-of-care analyser (Abbott; East Windsor, New Jersey, USA). Evaluation of the study participant was performed immediately on completion of stage 5 before post-ace hydration could occur. We assessed protocol adherence by inspecting the plastic bag for remaining pills. If the participant had lost the bag, we assessed protocol compliance by interview. Both the point-of-care device and the digital scale were calibrated prior to taking measurements.

\section{Study outcomes}

The primary outcome measure of AKI was by the established RIFLE criteria for severity of AKI as 'risk' of injury defined as $1.5 \times$ estimated baseline $\mathrm{Cr}$ and 'injury', defined as $2 \times \mathrm{Cr}^{13}{ }^{14}$ These two categories of AKI were combined to calculate the total incidence of AKI. The application of a categorical analysis of AKI via the RIFLE criteria rather than analysis of $\mathrm{Cr}$ as a continuous variable allowed qualification of ibuprofen's effect on AKI in descriptive terms consistent with prior ultramarathon studies. $^{2-4}$

A baseline expected glomerular filtration rate (GFR) of $100 \mathrm{~mL} / \mathrm{min}$ was used when age was <40 years, and 140 minus age when 40 years or older. ${ }^{15}$ This estimated GFR was then used in the 'modification of diet in renal disease' equation to back-calculate an estimated baseline $\mathrm{Cr},{ }^{16}$ with a numerator of 175 used for improved accuracy of the equation in a healthy population. ${ }^{17}$ All participants who took supplemental NSAID or less than three study pills were considered to be non-compliant with the study protocol. For the intent-to-treat (ITT) analysis, all randomised participants except those who did not have a $\mathrm{Cr}$ measurement for primary study outcome were analysed in the group they were originally randomised to, regardless of compliance.

Secondary outcome measures were detailed prior to trial initiation and included incidence of exercise-associated hyponatremia $(\mathrm{EAH})$ and Borg score survey for rating of perceived exertion at the fifth stage finish line as well as severity of AKI and change in Cr. The serum sodium cut-off points were the following: hypernatremia $>145 \mathrm{mmol} / \mathrm{L}$, normonatremia 135 $-<145 \mathrm{mmol} / \mathrm{L}$, hyponatremia $130-<135 \mathrm{mmol} / \mathrm{L}$ and severe hyponatremia $<130 \mathrm{mmol} / \mathrm{L}$. Weight change cut-off points were overhydration with a weight gain of $>+0.0 \mathrm{~kg}$, euhydration $=$ weight change between $-0.01 \%$ and $-3.0 \%$, and dehydration $=$ weight change $>-3 \%$.

\section{Statistical analysis}

Estimated baseline renal function and subsequent AKI criteria were based on the assumption that healthy ultramarathon runners had normal renal function at the start of a race, ${ }^{34}$ and estimated GFR was accurate compared with calculated GFR under rest conditions in healthy subjects. ${ }^{18}$ Previous studies indicate that multistage ultramarathon runners experience non-statistically significant changes in $\mathrm{Cr}$ and sodium levels between pre-race levels and the start of successive stages of racing, allowing us to presume a normal baseline renal function at the start of stage $5 .^{2}$

The study was designed as a non-inferiority trial, conducted with the primary outcome of AKI incidence and the null hypothesis that ibuprofen was worse than placebo regarding its detrimental effect on renal function during an ultramarathon. Before the study, an ad hoc verbal survey of ultramarathon runners during RacingThePlanet Iceland 2013 was conducted. The consensus was that a difference of $<15 \%$ AKI incidence 
would be an acceptable margin to accept potential renal injury for perceived analgesic benefits, and a greater incidence of AKI would discourage NSAID use. So assuming a clinically significant increase of AKI of $15 \%$ as the non-inferiority threshold, with incidence of AKI of $60 \%$ and $\beta=20 \%$ and $\alpha$ (one-sided) $=0.05$, we estimated that enrolment of 115 total participants would allow us to reject the null hypothesis and conclude ibuprofen was not inferior to placebo and appropriate analgesia to ingest during an ultramarathon.

Outcomes were analysed in the ITT population in order to evaluate real-world race conditions. Additionally, we analysed per-protocol results for non-inferiority for both the primary and secondary outcomes to assess the effect of a complete treatment course. ITT analyses of univariate comparisons were performed with Pearson's $\chi^{2}$ and Fisher's exact tests for categorical variables, independent-sample t-tests for continuous variables and Wilcoxon rank-sum test for non-normally distributed variables. Logistic regression was used to examine multivariate risk factors that had significance of $\propto<0.10$ for ORs. Pearson's correlation test was used to assess linear correlation between drug use (ibuprofen or placebo) and $\mathrm{Cr}$ changes from estimated to measured values to provide further insight into the different relationship in the two drugs. A one-sided Welch two-sample t-test was used to identify significant differences in measured $\mathrm{Cr}$ between treatment arms. p Values $<0.05$ were considered significant and 95\% CIs used. All analyses were

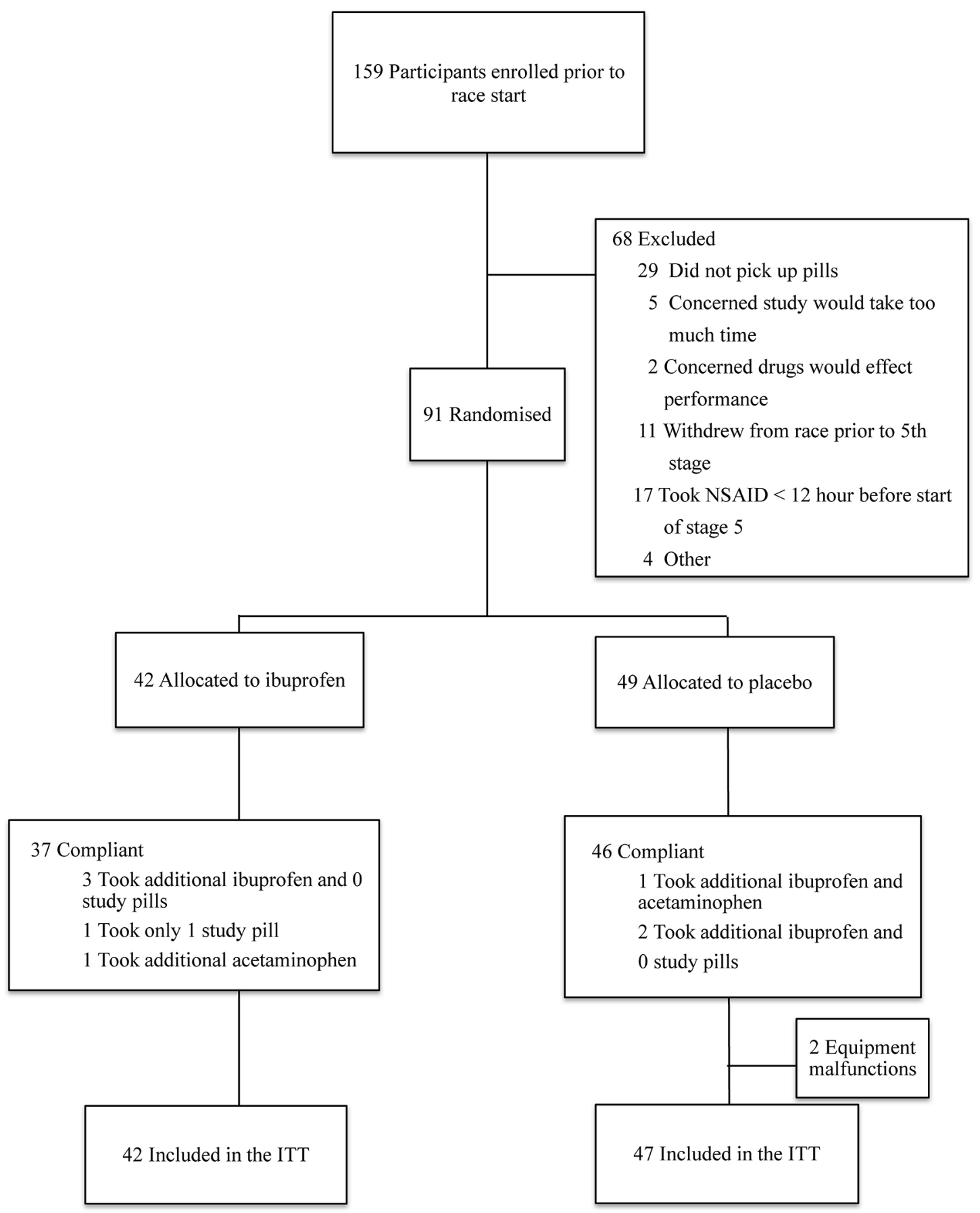

Figure 1 Consolidated Standards of Reporting Trials flow diagram. NSAID, non-steroidal anti-inflammatory drug. 
Table 1 Participant characteristics

\begin{tabular}{|c|c|c|c|}
\hline Characteristics & $\begin{array}{l}\text { Placebo } \\
\mathrm{n}=47(53 \%)\end{array}$ & $\begin{array}{l}\text { Ibuprofen } \\
\mathrm{n}=42(47 \%)\end{array}$ & $\begin{array}{l}\text { Difference between } \\
\text { treatment groups } \\
\text { p Value }\end{array}$ \\
\hline Female sex (n (\%)) & $34(72.3)$ & $33(78.6)$ & 0.66 \\
\hline Age, mean (SD) & $41.4(10.5)$ & $39.8(7.1)$ & 0.42 \\
\hline Body mass index $\left(\mathrm{kg} / \mathrm{m}^{2}\right)$, mean (SD) & $23.8(2.5)$ & $23.3(2.2)$ & 0.33 \\
\hline Baseline pack weight $(\mathrm{kg})$, mean (SD) & $10.2(2.2)$ & $10.0(2.1)$ & 0.62 \\
\hline Prior marathons, median ( $25 \%-75 \% \%$ IQR) & $4(0.5-11)$ & $4.5(0.25-10)$ & 0.86 \\
\hline Prior ultramarathons, median ( $25 \%-75 \% \%$ IQR) & $2(1-5.5)$ & $3.5(1.0-7.0)$ & 0.55 \\
\hline $\begin{array}{l}\text { Non-steroidal anti-inflammatory drug use prior to race, } \mathrm{n}(\%) \\
0-5 \times / \text { month }\end{array}$ & $44(93.6)$ & $36(85.7)$ & 0.38 \\
\hline $6-10 \times /$ month & $2(4.2)$ & $4(9.5)$ & 0.57 \\
\hline $11-15 \times /$ month & $1(2.1)$ & $0(0)$ & - \\
\hline $16-20 \times /$ month & $0(0)$ & $2(4.8)$ & - \\
\hline Finishing quintile & $2.9(1.4)$ & $2.5(1.3)$ & 0.18 \\
\hline Weight change $(\mathrm{kg})$ & $1.1(2.0)$ & $1.2(1.5)$ & 0.70 \\
\hline Number of ingested pills, median ( $25 \%-75 \% \%$ IQR) & $3(3-4)$ & $3(3-4)$ & 0.31 \\
\hline Creatinine baseline & $0.83(0.1)$ & $0.83(0.1)$ & 0.91 \\
\hline
\end{tabular}

conducted using computerised software ( $\mathrm{R} 3.1 .0$ software; Vienna, Austria).

\section{RESULTS}

Of 159 enrolled participants, 91 (57\%) were randomised to either ibuprofen or placebo and received the medications, with 89 participants (47\% ibuprofen and 53\% placebo) analysed for the study primary endpoint and who qualified for the ITT population (figure 1). The most common reasons for study exclusion included $43 \%$ who did not pick up the study medications, $25 \%$ ingested NSAID $<12$ hours prior to the start of stage 5 and another $16 \%$ who withdrew from the event prior to stage 5 . Two participants were excluded due to equipment malfunction as study end-of-stage $\mathrm{Cr}$ levels could not be analysed. Baseline demographics of the ITT population are described in table 1, with characteristics that were not significantly different between treatment arms $(p>0.1)$. The average amount of ibuprofen ingested was $1200 \mathrm{mg}$, with $75 \%$ of participants taking between 1200 and $1800 \mathrm{mg}$.

The overall incidence of AKI was 44\%. For the primary outcome in ITT analysis, ibuprofen increased the incidence of AKI by $18 \%$ (95\% CI $-4 \%$ to $41 \%)$, with a number needed to harm of 5.5. There was a statistically significant correlation between ibuprofen use and $\mathrm{Cr}$ change $\left(\mathrm{r}^{2}=0.2, \mathrm{p}<0.01\right)$ compared with placebo $\left(\mathrm{r}^{2}=0.05, \mathrm{p}=0.14\right)$ (figure 2$)$.

In the secondary outcomes, there was a non-statistically significant greater severity of AKI in the ibuprofen group, with $13 \%$ more meeting risk criteria $(95 \% \mathrm{CI}-9 \%$ to $34 \%)$ and $6 \%$ more meeting injury criteria $(95 \% \mathrm{CI}-10 \%$ to $21 \%$ ) (table 2 ). Although AKI was different between the groups, there was not a significant difference in post-race $\mathrm{Cr}$ between the two groups $(p=0.25,95 \% \mathrm{CI}-0.13$ to $\infty)$. There were no statistically significant differences between intervention groups and incidence of EAH and Borg scale, although decreased sodium (increased EAH) was found in the NSAID group by $8 \%$ (table 2 ). Incidence and severity differences per treatment groups were similar in the per-protocol population (data not shown).

Slower finishers by quintile were less likely to encounter AKI (OR $0.67,95 \%$ CI 0.47 to 0.98 ), and each quintile of slower finishing performance reduced the odds of AKI by 0.37 . Greater weight loss was significantly associated with AKI, in both absolute loss $(1 \mathrm{~kg}, \mathrm{p}<0.02)$ and percentage change $(1.3 \%, \mathrm{p}<0.002$, OR $1.2,95 \%$ CI 1.00 to 1.63$)$, where each $1 \%$ of weight loss increased the odds of AKI by 0.21 . There were no significant effects on the primary outcome of AKI incidence between treatment groups and the prespecified

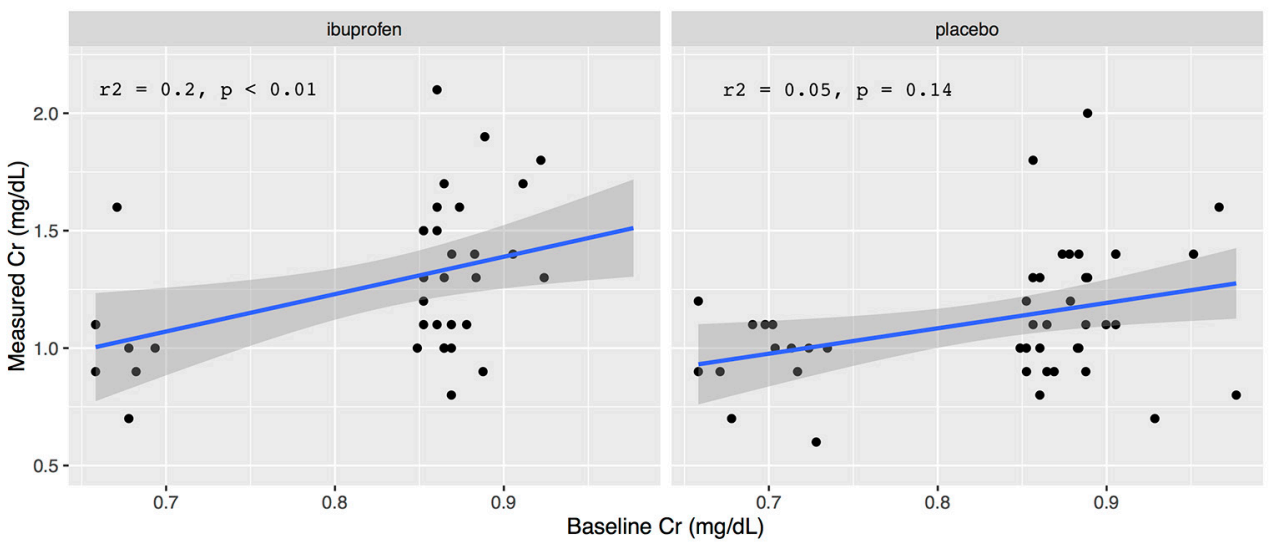

Figure 2 Correlation between changes in creatinine $(\mathrm{Cr})$ per treatment group. 
Table 2 Primary and secondary outcomes

\begin{tabular}{|c|c|c|c|c|c|}
\hline Outcomes & $\begin{array}{l}\text { Placebo } \\
n=47(53 \%)\end{array}$ & $\begin{array}{l}\text { Ibuprofen } \\
\mathrm{n}=42(47 \%)\end{array}$ & $\begin{array}{l}\text { Differences } \\
\%(95 \% \mathrm{Cl})\end{array}$ & $\mathrm{p}$ Value & $\begin{array}{l}\text { Differences } \\
\text { OR }(95 \% \mathrm{Cl})\end{array}$ \\
\hline Acute kidney injury incidence (\%) & $16(34 \%)$ & $22(52 \%)$ & $18(-4 \%$ to $41 \%)$ & 0.13 & 2.1 (0.9 to 5.1$)$ \\
\hline Risk (\%) & $12(25.5)$ & $16(38.1)$ & $12.6(-8.9 \%$ to $34.1 \%) \%)$ & 0.3 & 1.8 (0.7 to 4.5$)$ \\
\hline Injury (\%) & $4(8.5)$ & $6(14.3)$ & $5.8(-9.7 \%$ to $21.3 \%) \%)$ & 0.6 & $1.8(0.5$ to 7.7$)$ \\
\hline Exercise-associated hyponatremia (\%) & $7(7.3)$ & $3(14.8)$ & $7.5(-22.8 \%$ to $7.6 \%) \%)$ & 0.4 & 0.6 (0.1 to 2.3$)$ \\
\hline Borg (mean, SD) & $15.96(2.6)$ & $15.99(2.5)$ & $0.1(1 \%$ to $1.1 \%)$ & 1 & - \\
\hline
\end{tabular}

co-variates of the study participants with logistic regression (table 3).

\section{DISCUSSION}

This was the first double-blind randomised placebo-controlled trial to date that examined the causative effect of ibuprofen on AKI in ultramarathon runners. We found that runners who ingested an average of $1200 \mathrm{mg}$ ibuprofen over the course of a 50 -mile $(80 \mathrm{~km})$ foot race had a greater absolute rate of AKI compared with placebo. The severity of AKI was also worse in the ibuprofen group than the placebo group. Ibuprofen did not definitively demonstrate inferiority to placebo although it increased AKI by $18 \%$, crossing the prespecified non-inferiority threshold of $15 \%$. This approached, but did not reach, statistical significance by a small margin. Our study did not reach the enrollment threshold powered to establish non-inferiority and had a high rate of attrition, which may have led to a type II error. However, as the upper 95\% confidence limit for the primary outcome was $41 \%$ that far exceeded the non-inferiority margin, it is unlikely that the null hypothesis would be rejected and non-inferiority established with a larger cohort. As this trial was $40 \%$ larger than the only other prospective study on this subject, ${ }^{12}$ larger investigations into renal function and NSAID use of endurance runners may be logistically unfeasible.

We chose to power our study to a threshold effect size of 15 percentage points as that would alter the behaviour of the athletes who routinely take this medication and encounter AKI. Healthy population-based studies have found the risk of symptomatic acute renal disease caused by NSAID to be 1:100 000, spontaneously occurring in approximately $1 \%-5 \%$ of NSAID users that may translate to upwards of 2.5 million adverse events annually. ${ }^{19} 20$ Extrapolating the potential for symptomatic renal injury from NSAID use in the general population to high-risk endurance runners, we feel the risks outweigh the benefits of NSAID use and alternative options for analgesia such as acetaminophen should be considered.

NSAIDs inhibit cyclooxygenase, which prevents the breakdown of arachidonic acid to prostaglandins that contribute

\begin{tabular}{|c|c|c|}
\hline Participant characteristics & $\mathrm{p}$ Value & $\begin{array}{l}\text { Differences } \\
\text { OR }(95 \% \mathrm{Cl})\end{array}$ \\
\hline Age & 0.3 & 1.06 (0.57 to 1.92$)$ \\
\hline Body mass index $\left(\mathrm{kg} / \mathrm{m}^{2}\right)$ & 0.94 & 1.01 (0.78 to 1.32$)$ \\
\hline Pack weight (kg) & 0.64 & 1.09 (0.84 to 1.41$)$ \\
\hline Prior ultramarathons & 0.87 & $0.95(0.85$ to 1.05$)$ \\
\hline Prior marathons & 0.53 & $1.01(0.97$ to 1.05$)$ \\
\hline $\begin{array}{l}\text { Non-steroidal anti-inflammatory drug use/ } \\
\text { month prior }\end{array}$ & 0.34 & $2.0(0.06$ to 73.36$)$ \\
\hline
\end{tabular}

to renal vasodilation. Without these prostaglandins, there is reduced renal perfusion leading to impaired GFR. The injurious effects of renal hypoperfusion in endurance athletes may be exacerbated by rhabdomyolysis and resultant myoglobinaemia, a common occurrence in ultramarathoners whose average creatine kinase can range from $13000 \mathrm{U} / \mathrm{L}$ to $>40000$ UL. ${ }^{1021}$ Our study attempted to evaluate the clinical impact of NSAID by Borg scale, which is a validated scale of perceived exertion to quantify the physiological impact of exercise. ${ }^{22} 23$ As athletes ingest NSAID while exercising to minimise pain and optimise performance, it was hypothesised that the ingested drug could decrease perceived exertion secondary to decreased impact of pain. While there was no difference in the cohorts and perceived exertion by the Borg scale, figure 3 shows a non-significant increased Borg score in those with AKI which may reflect subtle effects from AKI on perceived exertion.

Greater weight loss and finishing performance were both found to be independent risk factors for the development of AKI. The correlation between weight loss as a proxy for dehydration and kidney injury has been shown in prior ultramarathon studies, ${ }^{3-5}$ which supports the deleterious effects of underhydration on renal perfusion. The slowest ultramarathon finishers have been found to be to $82 \%$ less likely than top finishers to encounter AKI, and slower finishing times may allow for greater hydration and minimise dehydration-induced kidney insult. ${ }^{3}$ As we were unable to quantify the amount of fluid ingested, the pathophysiology of dehydration and AKI could not be explored in this study.

There has been concern about the causative role of NSAID in $\mathrm{EAH}$, although reports are conflicted and of questionable clinical significance. ${ }^{24} \mathrm{EAH}$ is recognised as a relatively common issue in endurance running events. While EAH is usually an asymptomatic biochemical diagnosis that most often manifests as non-specific symptoms, it is a potentially fatal disease. NSAIDs act as an antidiuretic during exercise through inhibition of prostaglandins, sodium excretion and free water clearance that may contribute to a dilutional hyponatremia. Similar to our findings, the one other prospective trial that examined NSAID use and EAH showed no effect on post-race sodium levels compared

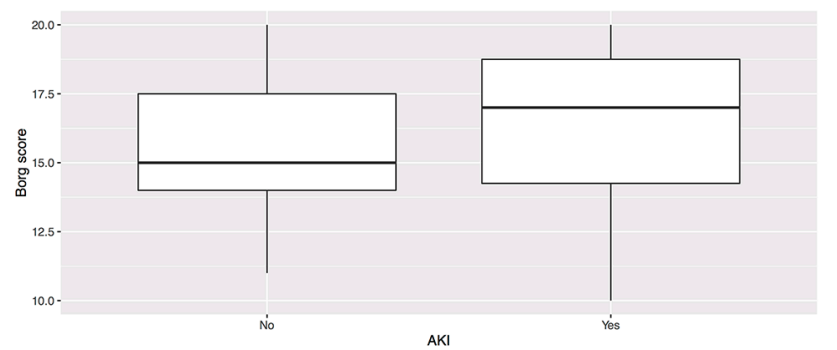

Figure 3 Borg score of perceived exertion and acute kidney injury (AKI). 
with placebo. ${ }^{12}$ Future prospective studies may help elucidate the effect of NSAID on EAH. Hydration in endurance events should be dictated by a 'drink to thirst' strategy, and the theoretical benefits of avoiding dehydration to minimise AKI should not be confused with overhydration, which should be avoided and is a risk factor for EAH morbidity and mortality.

This trial has several limitations. The average amount and rate of ibuprofen taken in our study in addition to distance covered may not be similar to other activities. However, as the observed total amount of NSAID ingested as well as distance run were both lower than the other prospective study which found no elevated creatinine in their cohort, ${ }^{12}$ we feel the results are likely generalisable to other events. Unfortunately, we were unable to gather baseline serum values and had to estimate the renal function to calculate AKI. Otherwise, the study would not have been feasible due to the $5-10 \mathrm{~min}$ required per participant to obtain and analyse each individual blood sample immediately prior to the race start. Estimating normal baseline serum electrolyte values in healthy runners is a well-established practice in ultramarathon research. ${ }^{2-4}$ It was unlikely there were spuriously elevated values from using an estimated baseline creatinine from the beginning of a mid-race stage as these $\mathrm{Cr}$ have been found to be similar to measured pre-race levels. ${ }^{2}$ Data from the four events were combined into a single cohort for analysis as each race had similar lengths and demands and grouped data between events is a common practice. ${ }^{325}$

\section{CONCLUSION}

This double-blind randomised placebo-controlled trial showed increased rates of AKI in those who took ibuprofen, and although not statistically inferior to placebo by a small margin, there was a number needed to harm of 5.5 people to cause one case of AKI. Consideration should therefore be taken before ingesting NSAID during endurance running as it could exacerbate renal injury.

Acknowledgements The authors acknowledge the generous support of RacingThePlanet staff and competitors, whose participation and enthusiasm made this study feasible. This work was presented at the American College of Emergency Physicians Scientific Assembly, October 2016, Las Vegas, Nevada, USA.

Contributors GSL had full access to all the data in the study and takes full responsibility for the integrity of the data and the accuracy of the data analysis. GSL and $\mathrm{CP}$ were responsible for data management, analysis and interpretation of the data, and statistical analysis. Study concept and design: GSL, BJK, KS. Acquisition, analysis or interpretation of data, and drafting of the manuscript: all authors. Obtained funding: GSL. Study supervision: GSL, KS.

Competing interests None declared.

Patient consent Obtained.

Ethics approval Stanford University School of Medicine.

Provenance and peer review Not commissioned; externally peer reviewed.

Data sharing statement There is no additional unpublished data from this study that has been made available.

(c) Article author(s) (or their employer(s) unless otherwise stated in the text of the article) 2017. All rights reserved. No commercial use is permitted unless otherwise expressly granted.

\section{REFERENCES}

12016 state of the Sport - U.S. Road Race Trends. secondary 2016 state of the Sport - U.S. Road Race Trends. 2016 http://www.runningusa.org/state-of-sport-us-trends2015? returnTo=annual-reports

2 Lipman GS, Krabak BJ, Waite BL, et al. A prospective cohort study of acute kidney injury in multi-stage ultramarathon runners: the Biochemistry in Endurance Runner Study (BIERS). Res Sports Med 2014;22:185-92.

3 Lipman GS, Krabak BJ, Rundell SD, et al. Incidence and prevalence of acute kidney Injury during Multistage Ultramarathons. Clinical journal of sport medicine: official journal of the Canadian Academy of Sport Medicine 2015.

4 Hoffman MD, Stuempfle KJ, Fogard K, et al. Urine dipstick analysis for identification of runners susceptible to acute kidney injury following an ultramarathon. J Sports Sci 2013:31:20-31.

5 Kao WF, Hou SK, Chiu YH, et al. Effects of 100-km ultramarathon on acute kidney injury. Clin J Sport Med 2015;25:49-54.

6 Reid SA, King MJ. Serum biochemistry and morbidity among runners presenting for medical care after an Australian mountain ultramarathon. Clin I Sport Med 2007; 17:307-10

7 Seedat YK, Aboo N, Naicker S, et al. Acute renal failure in the "Comrades Marathon" runners. Ren Fail 1989;11:209-12.

8 Bruso JR, Hoffman MD, Rogers IR, et al. Rhabdomyolysis and hyponatremia: a cluster of five cases at the $161-\mathrm{km} 2009$ Western States Endurance Run. Wilderness Environ Med 2010;21:303-8.

9 Irving RA, Noakes TD, Burger SC, et al. Plasma volume and renal function during and after ultramarathon running. Med Sci Sports Exerc 1990;22:581-7.

10 Nieman DC, Henson DA, Dumke CL, et al. Ibuprofen use, endotoxemia, inflammation, and plasma cytokines during ultramarathon competition. Brain Behav Immun 2006;20:578-84.

11 Hoffman MD, Fogard K. Factors related to successful completion of a $161-\mathrm{km}$ ultramarathon. Int I Sports Physiol Perform 2011;6:25-37.

12 Dumke CL, Nieman DC, Oley K, et al. Ibuprofen does not affect serum electrolyte concentrations after an ultradistance run. Br J Sports Med 2007;41:492-6.

13 Lewington AJ, Sayed A. Acute kidney injury: how do we define it? Ann Clin Biochem 2010:47:4-7.

14 Venkataraman R, Kellum JA. Defining acute renal failure: the RIFLE criteria. J Intensive Care Med 2007;22:187-93.

15 Granerus G, Aurell M. Reference values for 51Cr-EDTA clearance as a measure of glomerular filtration rate. Scand I Clin Lab Invest 1981;41:611-6.

16 National Kidney Foundation. K/DOQI clinical practice guidelines for chronic kidney disease: evaluation, classification, and stratification. Am J Kidney Dis 2002;39(2 Suppl 1):S1-266.

17 Levey AS, Coresh J, Greene T, et al. Expressing the Modification of Diet in Renal Disease Study equation for estimating glomerular filtration rate with standardized serum creatinine values. Clin Chem 2007;53:766-72.

18 Poortmans JR, Gulbis B, De Bruyn E, et al. Limitations of serum values to estimate glomerular filtration rate during exercise. Br J Sports Med 2013;47:1166-70.

19 Beard K, Lawson DH, MacFarlane GJ. Non-steroidal anti-inflammatory drugs and acute renal disease: a case control study. Pharmacoepidemiology \& Drug Safety 1992;1:3-9.

20 Whelton A. Nephrotoxicity of nonsteroidal anti-inflammatory drugs: physiologic foundations and clinical implications. Am J Med 1999;106:13S-24.

21 Hoffman MD, Ingwerson JL, Rogers IR, et al. Increasing creatine kinase concentrations at the $161-\mathrm{km}$ Western States Endurance Run. Wilderness Environ Med 2012;23:56-60.

22 Scherr J, Wolfarth B, Christle JW, et al. Associations between Borg's rating of perceived exertion and physiological measures of exercise intensity. Eur J Appl Physiol 2013;113:147-55.

23 Chen MJ, Fan X, Moe ST. Criterion-related validity of the Borg ratings of perceived exertion scale in healthy individuals: a meta-analysis. I Sports Sci 2002:20:873-99.

24 Wharam PC, Speedy DB, Noakes TD, et al. NSAID use increases the risk of developing hyponatremia during an Ironman triathlon. Med Sci Sports Exerc 2006; 38:618-22

25 Hoffman MD, Hew-Butler T, Stuempfle KJ. Exercise-associated hyponatremia and hydration status in 161-km ultramarathoners. Med Sci Sports Exerc 2013;45:784-91. 